\title{
Intelligently optimized electrospun polyacrylonitrile/poly(vinylidene fluoride) nanofiber: Using artificial neural networks
}

\author{
M. M. Salehi ${ }^{1}$, F. Hakkak ${ }^{2}$, S. M. Sadati Tilebon ${ }^{3}$, M. Ataeefard ${ }^{4}$, M. Rafizadeh ${ }^{2}$ \\ ${ }^{1}$ Formulation and Development of Applications of Chemical and Polymeric Compounds Research Group, Chemical, \\ Polymeric and Petrochemical Technology Development Research Division, Research Institute of Petroleum Industries, \\ Tehran, Iran \\ ${ }^{2}$ Polymer Engineering and Color Technology, Amirkabir University of Technology, P.O. Box 15875-4413, Tehran, Iran \\ ${ }^{3}$ Iran University of Science and Technology, school of chemical, petroleum, and gas engineering, Narmak, Tehran, Iran \\ ${ }^{4}$ Department of Printing Science and Technology, Institute for Color Science and Technology, Tehran, Iran
}

Received 27 August 2019; accepted in revised form 28 March 2020

\begin{abstract}
In this work, an intelligent approach is applied for the first time in the modelling and optimization of electrospun Polyacrylonitrile/Poly(Vinylidene Fluoride) (PAN/PVdF) nanofiber properties. A genetic algorithm-based computer code was developed to optimize the architecture of an artificial neural network, by which an accurate model was developed for the prediction of nanofiber diameter, the standard deviation of nanofiber diameter and porosity of electrospun membrane. Electrospinning of polyacrylonitrile/poly(vinylidene fluoride) (PAN/PVdF) was applied to obtain a quantitative relationship between selected electrospinning parameters (namely applied voltage, solution concentration, and PVdF composition) and nanofiber diameter, the standard deviation of nanofiber diameter and porosity of electrospun membrane. The morphology and nanofiber diameter were investigated by field emission scanning electron microscopy (FESM). The range of produced nanofiber diameters was from 116 to $379 \mathrm{~nm}$. It seemed that the nanofiber diameter and standard deviation of nanofiber diameter decrease with PVdF composition and increase with solution concentration. The applied voltage had no important effect on the nanofiber diameters. The porosity of the electrospun membrane decreases with solution concentration and increases with PVdF composition.
\end{abstract}

Keywords: polymer composites, artificial intelligence, genetic algorithm-based computer code, nanofiber diameter, electrospinning

\section{Introduction}

Nanofibers are fibers with a diameter in the range of nanometer. Nanofibers show excellent properties such as very small nanofiber diameters, consequently large surface area per mass ratio, high porosity and small pore sizes, appreciable mechanical and electrochemical properties. These properties lead to their excellent application in many areas such as nanocomposites, drug delivery, tissue engineering, sensors, filters, and polymer electrolyte in batteries $[1,2]$.
Jian et al. [3] prepared PAA nanofibers with diameters ranging from hundreds of nanometers down to sub-nanometer via electrospinning from a polyamic acid (PAA) with ultrahigh molecular weight.

Nanofibers can be produced through different techniques such as phase separation, template synthesis, self-assembly, electrospinning, etc. Among these methods, electrospinning is the simple, low-cost, and most commonly used technique. In electrospinning, continuous ultrathin fibers with controllable 
nanometer diameters can be produced from various polymers.

Electrospinning is a flexible process, so in electrospinning, it is possible to control the shape and arrangement of the fibers for various application purposes. The instruments necessary for electrospinning include a high voltage supplier, a syringe with a small diameter needle, a syringe pump, and a metal collector. Duan et al. [4] applied coaxial electrospinning with air-blowing-assistance to improve the productivity of core/sheath and hollow fibers. Different core and shell materials were used for this electrospinning.

In electrospinning, a high voltage is applied between a droplet of polymer solution or melt at the tip of a syringe's needle and a grounded collector, which leads to the production of surface charge on the polymer solution. Increasing the applied voltage leads to the intensity increase of the electric field. By increasing the electric field, the domination of the electric force over the surface tension occurs, and consequently, a jet is ejected and accelerated toward the collector. On account of elongation, the solution jet rapidly thins and dries as a result of solvent evaporation. By decreasing the jet diameter, the surface charge density increases, which leads to the production of smaller jets due to high repulsive forces. Finally, solidification takes place, and nanofibers are collected on the surface of the collector as a random, nonwoven mat [5-8]. Ding et al. [9] applied theoretical deduction and experimental calculation to evaluate the velocities and draw ratios of the charged jets at different electrospinning stages. The theoretical calculation was compared with experimental data using polyamic acid as a model polymer for electrospinning.

Recently electrospun nanofibers have received significant attention to use as polymer electrolytes in lithium batteries. Ionic conductivity is one of the most important characteristics of polymer electrolytes. It was reported that in polymer electrolyte morphology of nanofibers has a substantial effect on their electrochemical properties. So decreasing the nanofiber diameter leads to higher porosity and electrolyte solution uptake and hence higher ionic conductivity [10]. Therefore, one of the most recent challenges of researchers is to decrease the nanofiber diameter by changing electrospinning parameters. Electrospinning parameters can be divided into three categories: processing conditions (applied voltage, volume flow rate, electric field strength, tip to collector distance, electrostatic field shape, needle diameter, etc.), solution properties (polymer molecular weight, viscosity, conductivity, concentration, solvent volatility, surface tension, dielectric constant, etc.), and ambient conditions (temperature, atmosphere pressure, humidity, air velocity in the electrospinning chamber, etc.) [11]. Therefore, introducing a known relationship among these parameters and nanofiber diameter is desired. Ribeiro et al. [12] systematically changed the processing parameters to analyze their influence on fiber morphology, fiber orientation, and on the crystallinity and crystal phase of the electroactive poly (vinylidene fluoride) electrospun membranes. Areias et al. [13] studied the relationship between processing parameters (solvent composition, polymer concentration in the solution, feeding rate, applied voltage, traveling distance between the needle, and the collector) and fiber morphology. They used Taguchi's methodology was to determine which parameters have the strongest influence on mean fiber diameter and fiber homogeneity.

Among different polymers, polyacrylonitrile (PAN) and poly(vinylidene fluoride) (PVdF) have received considerable attention as host polymers in polymer electrolytes. PVdF has a high dielectric constant and fluorine atoms in the backbone structure. These features are useful in dissociating the lithium salt to lithium ions while transforming into a polymer electrolyte [14-16]. PAN-based electrolytes have shown interesting characteristics like high ionic conductivity, thermal stability, electrolyte uptake, and compatibility with the lithium electrodes [17-19]. Moreover, PAN minimizes the formation of dendrite growth during the charging/discharging process of lithium batteries. Published results indicate that $-\mathrm{C}=\mathrm{N}$ groups in PAN could interact with $-\mathrm{C}=\mathrm{O}$ groups of the liquid electrolytes (E.C.) as well as with $\mathrm{Li}+$ ions [2023]. Hence, the polymer electrolytes prepared from composites of PAN and PVdF may have the synergistic advantages of both polymers.

In our previous studies, the authors used the response surface method (R.S. M) to create numerical models to capture the variation of PAN/PVdF nanofiber properties as a function of the variables above. Modeling of voltage, solution concentration, PVdF composition was first performed using RSM to obtain reduced models, and then we optimized these three variables to obtain minimum nanofiber diameter [24].

Though modeling/optimization technique used was successful in detecting and optimizing properties of 
PAN/PVdF nanofiber, the results could not be generalized to cases not being fed into the model. Moreover, predictions were merely reliable for scenarios received by the reduced model and could not imitate the real behavior of the system.

Normally, one may apply classical or stochastic modeling approaches for thorough identification of the behavior of a given system $[25,26]$. The use of artificial intelligence-based models instead of polynomial-based models may provide an opportunity to learn the behavior of a process, to generalize the behavior learned to experimental scenarios never been examined, and to find the best set of input variables needed for achieving the desired properties [27]. In the current work, an artificial intelligence approach was newly applied in detecting and identifying variation patterns of PAN/PVdF nanofiber properties (nanofiber diameter, the standard deviation of nanofiber diameter and porosity of membrane) to use as polymer electrolyte in lithium batteries. In this sense, an in-house computer code was developed combining artificial neural network (ANN), and genetics algorithm (G.A.) approaches. The code makes possible intelligent modeling and optimization of properties of PAN/PVdF nanofiber.

\section{Experimental}

\subsection{Materials}

$N, N$-dimethylformamide (DMF), PAN and PVdF (Solef 1010) were purchased from Merck Co. (Germany), Arak Petrochemical Inc. (Iran) and Solvay Co. (France) respectively.

\subsection{Solution preparation and electrospinning}

$\mathrm{PVdF}$ and PAN were dissolved in DMF at ambient conditions. All the polymer samples were dried at $60^{\circ} \mathrm{C}$ for $12 \mathrm{~h}$ before use.

A $1 \mathrm{ml}$ syringe was used as an injector. The needle size was $0.41 \mathrm{~mm}$. Electrospinning was carried out on a horizontal setup. The electrospinning setup consisted of a syringe and needle, a syringe pump (New Era Pump System, NE1000, USA), a grounded electrode (Aluminum sheet), and a high-voltage power supply (Gamma High Voltage Research, RR60, USA) that could generate positive D.C. voltages up to $60 \mathrm{kV}$.

After performing some preliminary experiments to obtain bead-free, stable, and continuous nanofibers, it was concluded that applied voltage, solution concentration, and PVdF composition were the most influential parameters. The parameters were chosen as follows:

applied voltage in the range of 19 to $23 \mathrm{kV}$; solution concentration in the range of 14 to $18 \mathrm{wt} \%$; $\mathrm{PVdF}$ composition in the polymer part of the solution of 25,50 , and $75 \mathrm{wt} \%$; distance between the tip of the spinneret and collector $17 \mathrm{~cm}$; and solution feed rate $200 \mu \mathrm{l} / \mathrm{h}$.

Electrospinning was performed at room temperature, and the syringe setup was enclosed in a chamber for adjusting and controlling the temperature.

\subsection{Morphology studies}

The morphology and microstructure of the PAN/ PVdF nanofibers were observed by FESEM (Phillips XL-30, Netherlands). The samples coated with gold for enhanced conductivity using SPI sputter coater. The diameter of the nanofibers was measured and analyzed by Image J software $1.43 \mathrm{u}$ (National Institutes of Health, USA). Generally, 8-10 images were taken for each sample.

\subsection{Porosity measurements}

Porosity $\%$ of PAN/PVdF membranes was determined using $n$-butanol uptake method. The $n$-butanol penetrates the pores of the membrane and occupies all the available pores, without interacting with the membrane, and thus gives a measure of the total pore volume in the material. In order to measure the porosity $\%$, membranes were immersed in n-butanol for $2 \mathrm{~h}$. The mass of PAN/PVdF membranes before and after absorption was measured. The porosity $\%$ of the membrane was calculated using the following Equation (1) [28]:

Porosity $\%=\frac{\frac{M_{\mathrm{BuOH}}}{\rho_{\mathrm{Bu} \mathrm{H}}}}{\frac{M_{\mathrm{m}}}{\rho_{\mathrm{BuOH}}}+\frac{M_{\mathrm{m}}}{\rho_{\mathrm{m}}}}$

where $M_{\mathrm{m}}$ the mass of dry membrane, $M_{\mathrm{BuOH}}$ is the mass of $n$-butanol absorbed, $\rho_{\mathrm{BuOH}}$ and $\rho_{\mathrm{p}}$ are the density of $n$-butanol and the polymer, respectively.

\section{Model development and optimization}

In this work, GA-based optimization was implemented to ANN models developed based on experimental data. In the first step of modeling with the artificial intelligence approach, we obtained ANN models. In the second step, models were applied in the optimization of PAN/PVdF properties based on a reciprocal intelligent tool. Three parameters 
containing applied voltage, solution concentration, and PVdF composition were selected as input variables to assess their effect on nanofiber diameter, the standard deviation of nanofiber diameter and porosity (output variables), individually and simultaneously. Accordingly, experiments were performed to obtain sufficient data to be fed into ANN models (Table 1). The training of ANN allows for learning the appropriate behavior of the selected property. The weights and biases of the ANN are to be found in a manner to minimize prediction error made by the network in the course of training $[29,30]$. When the training has been completed, ANNs are able to foresee PAN/PVdF nanofiber upon receiving any input similar to the pattern they are taught.

According to Table 1, different scenarios were feeding into ANN models assigned to each property of PAN/PVdF nanofiber. Hyperbolic tangent sigmoid activation function was employed to normalize input variables in $[-1,+1]$ interval [31] (Equation (2)):

$X_{\mathrm{i}}=2 \cdot \frac{x_{\mathrm{i}}-x_{\min }}{x_{\min }-x_{\max }}-1$

where $X_{\mathrm{i}}$ denotes a normalized value of input variable $x_{\mathrm{i}}$, while $x_{\min }$ is minimum and $x_{\max }$ is the maximum value of target functions, respectively [32].

An in-house code was developed in this work that can conceptually search for best configurations, i.e., for dividing data into 'training', 'validation', and 'test' sets. Accordingly, 75 percent of data was chosen to be fed to model for training. On the other hand, $15 \%$ of data were selected for validation of ANN model. Finally, other data were utilized for testing of configured ANN structure. Although there is no rigid rule to find the appropriate number of neurons in the hidden layers, the complexity of the relationship between inputs and outputs plays a key role $[33,34]$. Different combinations of neurons (one to ten neurons in each hidden layer) in two hidden layers were tested for choosing the best network with a minimized error. The function defined through Equation (2) is applied in output, and hidden layers were used as activation transfer function [32, 35] (Equation (3)):

$$
f(x)=\frac{\mathrm{e}^{\mathrm{x}}-\mathrm{e}^{-\mathrm{x}}}{\mathrm{e}^{\mathrm{x}}+\mathrm{e}^{-\mathrm{x}}}
$$

According to the flowsheet shown in Figure 1, a well-organized computer code was written to intelligently model properties of the electrospun nanofiber. To optimize parameters of the model, i.e., to find the best weights and biases that match the input and output variables, a gradient descent (G.D.) method. The number of neurons in hidden layer 1 (NH1) and hidden layer 2 (NH2) was varied in the range of 1-10 (maximum level of neurons in each hidden layer (max NH1 and max NH2) was considered equal to 10). The reliability of ANN model was

Table 1. Scenarios considered as internal data for the constructed model.

\begin{tabular}{|c|c|c|c|c|c|c|}
\hline \multirow[b]{2}{*}{ Scenario } & $1^{\text {st }}$ input, $X_{1}$ & $2^{\text {nd }}$ input, $X_{2}$ & $3^{\text {rd }}$ input, $X_{3}$ & $1^{\text {st }}$ output, $Y_{1}$ & $2^{\text {nd }}$ output, $Y_{2}$ & $3^{\text {rd }}$ output, $Y_{3}$ \\
\hline & $\begin{array}{c}\text { PVdF composition } \\
{[\%]}\end{array}$ & $\begin{array}{c}\text { Solution concentration } \\
{[\%]}\end{array}$ & $\begin{array}{c}\text { Voltage } \\
{[\mathrm{kV}]}\end{array}$ & $\begin{array}{c}\text { Porosity } \\
{[\%]}\end{array}$ & $\begin{array}{c}\text { Standard deviation } \\
{[\mathrm{nm}]}\end{array}$ & $\begin{array}{c}\text { Diameter } \\
{[\mathrm{nm}]}\end{array}$ \\
\hline 1 & 50 & 18 & 19 & 48.2 & 42.65 & 379 \\
\hline 2 & 50 & 16 & 21 & 74.4 & 24.97 & 176 \\
\hline 3 & 50 & 16 & 21 & 75.3 & 25.43 & 178 \\
\hline 4 & 75 & 18 & 21 & 62.1 & 30.06 & 246 \\
\hline 5 & 75 & 16 & 23 & 74.3 & 23.87 & 174 \\
\hline 6 & 50 & 16 & 21 & 75.5 & 24.27 & 179 \\
\hline 7 & 50 & 16 & 21 & 74.6 & 25.00 & 176 \\
\hline 8 & 50 & 18 & 23 & 57.3 & 37.56 & 290 \\
\hline 9 & 25 & 16 & 19 & 56.6 & 38.08 & 291 \\
\hline 10 & 25 & 18 & 21 & 53.2 & 40.97 & 312 \\
\hline 11 & 75 & 14 & 21 & 90.1 & 18.57 & 116 \\
\hline 12 & 25 & 14 & 21 & 68.4 & 26.75 & 219 \\
\hline 13 & 25 & 16 & 23 & 60.2 & 35.76 & 260 \\
\hline 14 & 50 & 14 & 19 & 86.6 & 20.68 & 135 \\
\hline 15 & 50 & 16 & 21 & 75.3 & 24.03 & 175 \\
\hline 16 & 75 & 16 & 19 & 82.7 & 22.34 & 149 \\
\hline 17 & 50 & 14 & 23 & 87.4 & 19.74 & 132 \\
\hline
\end{tabular}




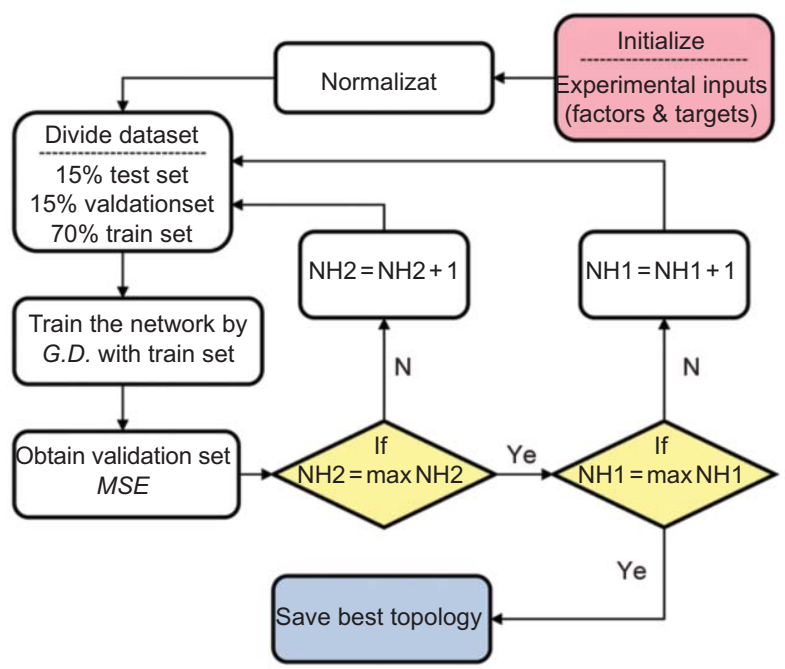

Figure 1. Modeling flowchart based on ANN method.

measured using mean squared error (MSE) criterion (Equation (4)):

$M S E=\frac{1}{n} \sum_{\mathrm{i}=1}^{\mathrm{n}}\left(Y_{\mathrm{i}}-\bar{Y}_{\mathrm{i}}\right)^{2}$

where $n$ is the number of samples, $Y_{\mathrm{i}}$ and $\bar{Y}_{\mathrm{i}}$ are experimental and predicted value of response for sample $i$.

In the light of the above procedure, one ANN model was yielded to predict of study effective parameters on PAN $\backslash P V d F$ nanofiber separately. In the next step, for the multi-objective optimization of the nanofiber production process, a Non-Dominated Sorting Genetic Algorithm-II (NSGA-II) was utilized. For the production of chromosomes, the value of each gene was randomly chosen in view of the values of variables given in Table 1. The code was then implemented to find the fitness of each chromosome to optimize nanofiber properties simultaneously. After giving pairs of chromosomes were compared together, they were isolated from the remainder, as the first Pareto front. This operation was surveyed to obtain Pareto fronts 2, 3, etc. Pareto front $\mathrm{n}$ was assigned to the chromosome that has been dominated $(n-1)$ times. We also used crowding distance (C.D.) values, as the second criterion for optimization, was to sort Pareto fronts (Equation (5)):

C.D. $(i)=\sum_{\mathrm{x}=1}^{\mathrm{N}} \frac{d_{\mathrm{x}}(i)}{\Delta_{\mathrm{x}}(i)}$

where $d_{\mathrm{x}}(i)=\left|F_{\mathrm{x}}(i+1)-F_{\mathrm{x}}(i-1)\right|$

and $\Delta_{\mathrm{x}}(i)=\left|\max F_{\mathrm{x}}-\min F_{\mathrm{x}}\right|$

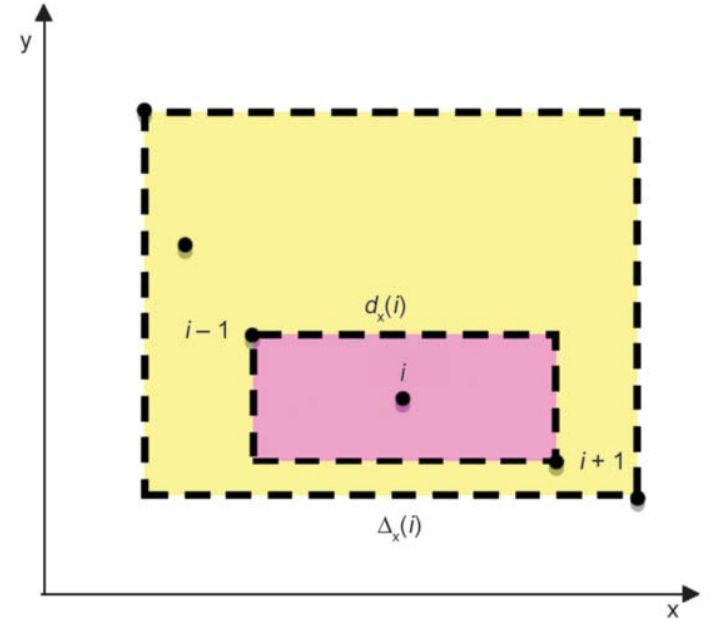

Figure 2. Crowding distance calculation parameters for sample $i$.

Table 2. The value of parameters used for multi-objective optimization by G.A. approaches.

\begin{tabular}{|l|l|}
\hline Optimization parameter & Value \\
\hline Initial population size & 30 \\
\hline Crossover mechanism & Arithmetic \\
\hline Crossover rate & $70 \%$ \\
\hline Mutation mechanism & Polynomial \\
\hline Mutation rate & $40 \%$ \\
\hline Selection mechanism & Ternary Tournament Selection \\
\hline
\end{tabular}

In the Equation (5), $N$ is the number of objective functions, C.D. $(i)$ is crowding distance of chromosome $i$, and $d_{\mathrm{x}}(i)$ and $\Delta_{\mathrm{x}}(i)$ based on the objective function $\mathrm{x}$ is shown in Figure 2. After estimating the extent of fitness based on primary and secondary constraints, chromosomes were sorted only by the most favorable to unfavorable order. The best chromosomes were

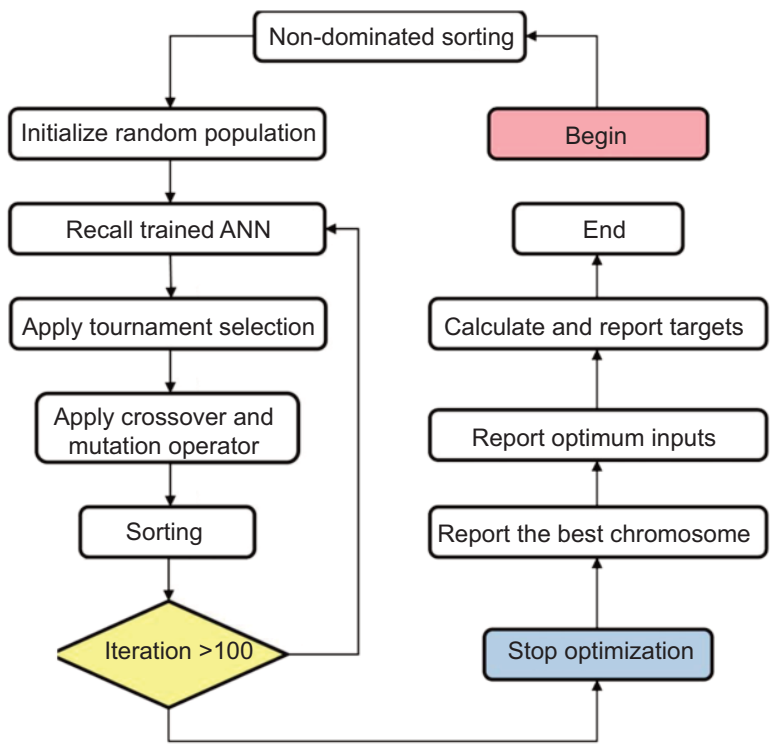

Figure 3. Flowchart of NSGA-II based optimization. 
chosen as the first Pareto front, followed by selecting, pairing, reproducing, and muting. Parents and children of the new generation were revisited for fitness utilizing a non-dominated sorting algorithm. Table 2 gives the values of parameters employed for the optimization of nanofiber properties on the ground of NSGA-II evolutionary algorithm (Figure 3).

\section{Results and discussion}

Normally, for nanofibrous polymer electrolyte production with suitable characteristics, nanofiber diameter and porosity of the nanofibrous membrane should be controlled. In the case of the electrospinning of PAN/PVdF nanofiber to the best of the authors' knowledge, there is no report on the optimization of the parameters above based on ANN. The effect of voltage, solution concentration, and PVdF composition used as the main material parameters to determine nanofiber diameter, the standard deviation
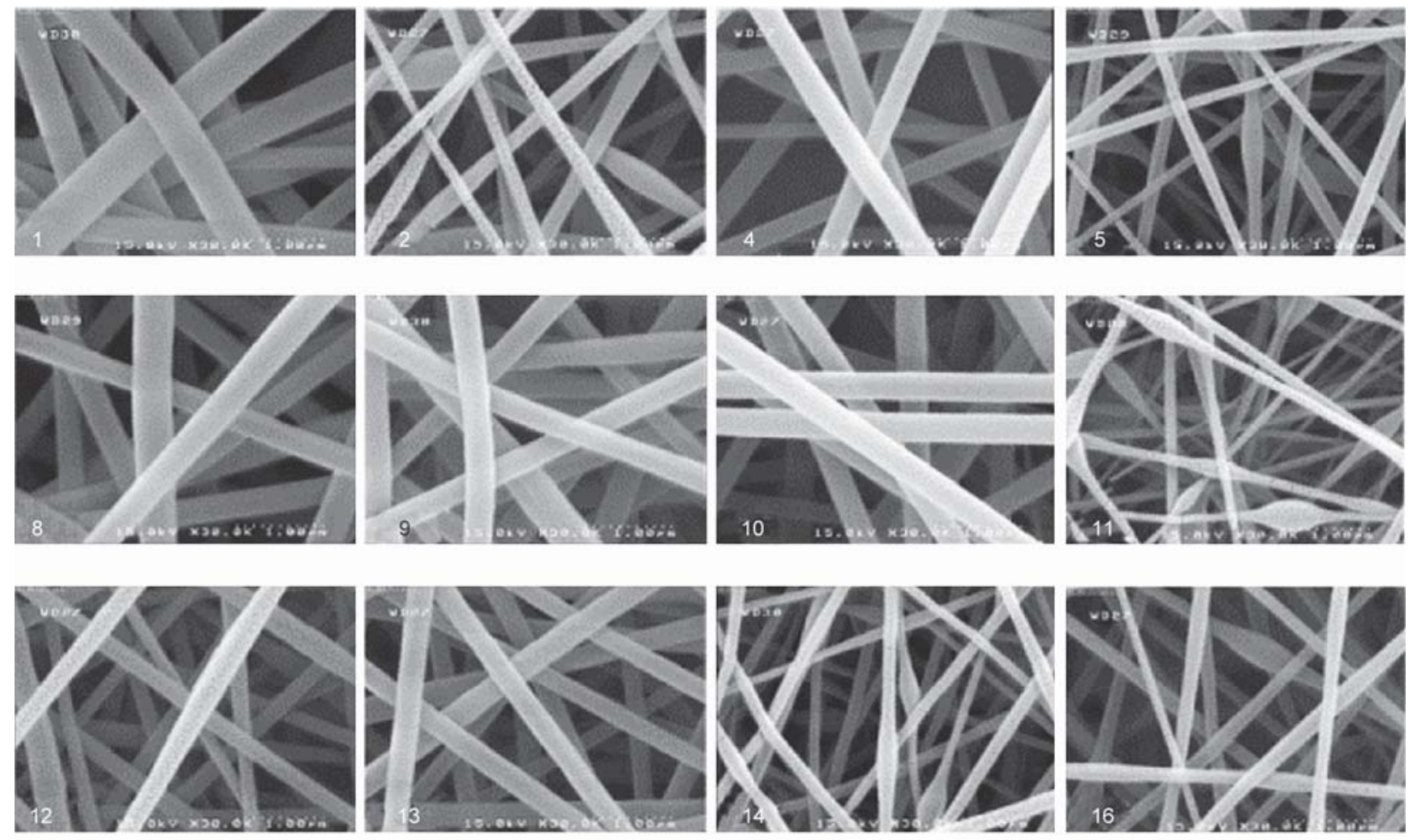

of nanofiber diameter, and the porosity of electrospun PAN/PVdF nanofibrous membrane with ANN.

Figure 4 shows SEM images for all samples. As it can be seen that bead-free and continuous nanofibers were obtained in all experiments. These images are in accordance with the results of the modeling and theoretical approach.

In this study, to unveil the complex nature of the relationship between inputs and outputs, an artificial neural network was used. The optimized structure of ANN with 5 neurons in each of hidden layer 1 and hidden layer 2 was achieved (Figure 5). Selecting of best ANN structure in this study was done by considering the validation step MSE. Between the different probable networks with 1 to 10 neurons in each hidden layer, a combination of 5 neurons in hidden layer 1 and 5 neurons in hidden layer 2 was introduced as the best structure. This network has a 3 level input $\left(X_{1}, X_{2}\right.$, and $\left.X_{3}\right)$ and 3 outputs $\left(Y_{1}, Y_{2}\right.$, and $\left.Y_{3}\right)$.

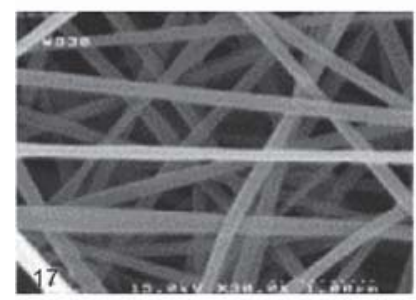

Figure 4. SEM micrographs of electrospun PAN/PVdF nanofibers (based on Table 1). Scale bars represent $1 \mu \mathrm{m}$. 


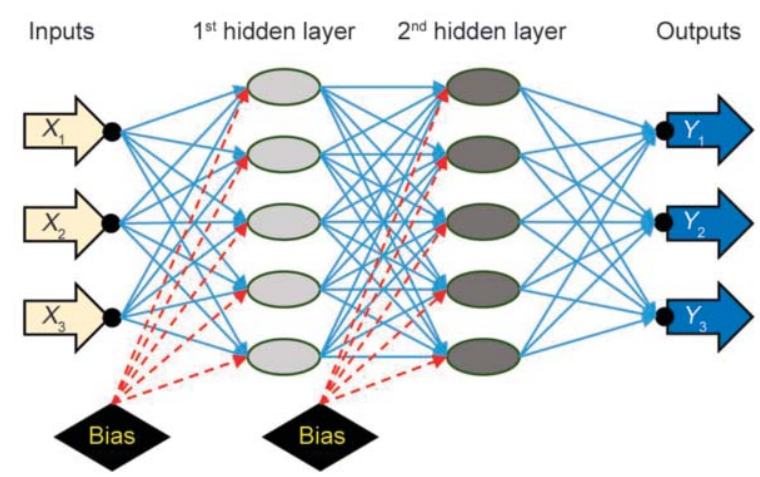

Figure 5. Optimized structure of ANN.

\subsection{Modeling of PAN/PVdF nanofiber production with ANN}

The performance of the developed ANN model is further checked by comparing the experimental and predicted values of each response variable. From Figure 6, errors in the prediction of each response are minor, and these figures show that ANN-based

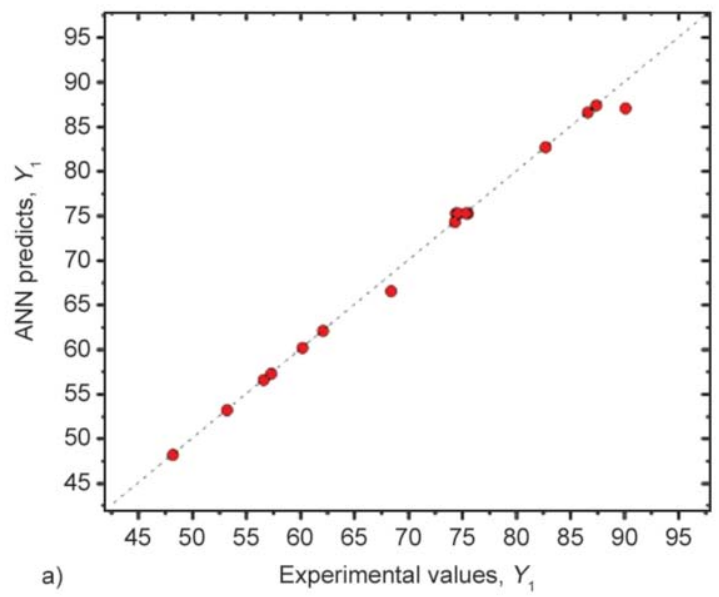

predicted $Y_{1}, Y_{2}$, and $Y_{3}$ are really close to the experimental values. Significant similarities between the ANN model outputs and experimental results show good accuracy of model predictions. From the statistical point of view, the slope and intercept of the regression equations for the outputs are very close to 1 and 0 , respectively. Furthermore, modeling accuracy based on $R$ calculation was calculated at about 0.99 for training, validation, and testing sections. Finally, $R$ for all experimental data compared with modeling results was achieved about 0.9991 .

To get a brighter prospect of the accuracy of the optimized ANN developed in this work, the quantities of target functions for the whole trained, validated, and tested data sets are compared in Figure 5. The plots show that the optimized ANN produces good predictions for each response. Besides, prediction error (based on Equation (6)) in each sample is shown in Figure 7 that it is clarified that ANN-model has a very suitable prediction for $Y_{1}, Y_{2}$, and $Y_{3}$ :

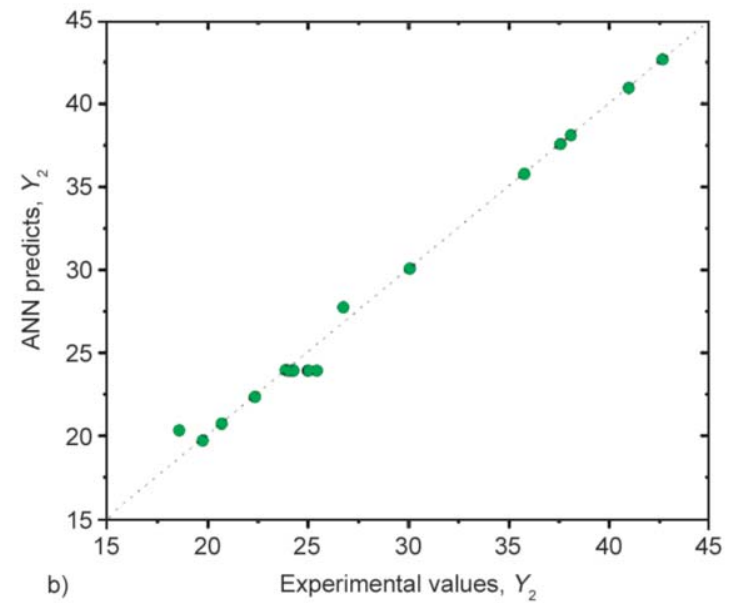

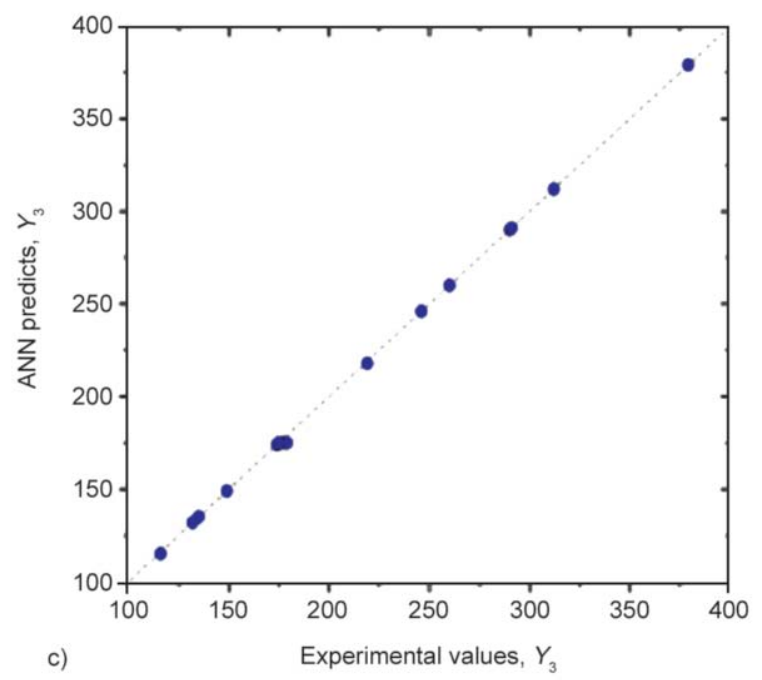

Figure 6. Relationship between the experimental data and the optimized ANN model outputs. a) Porosity [\%], b) standard deviation $[\mathrm{nm}], \mathrm{c})$ diameter $[\mathrm{nm}]$. 

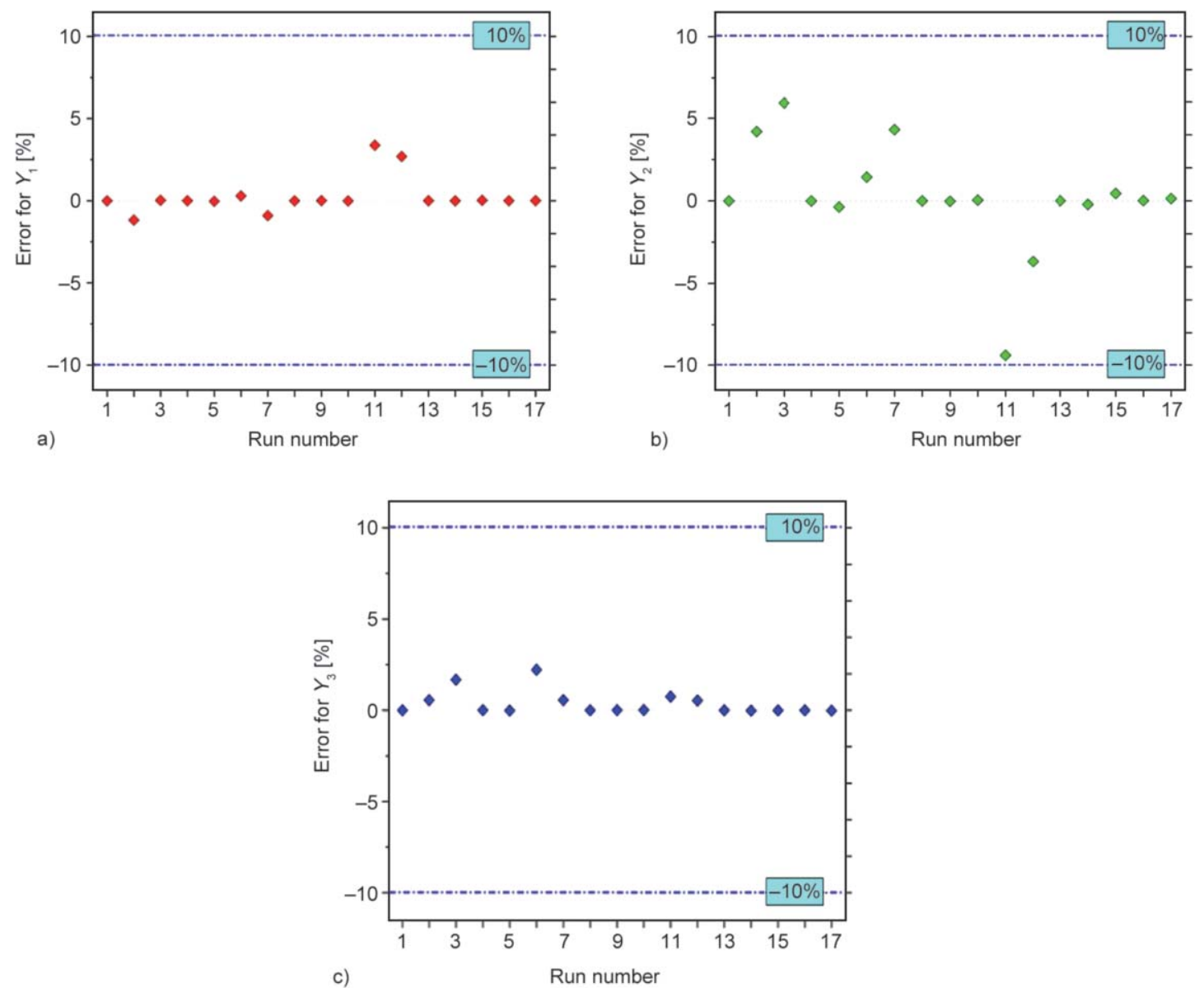

Figure 7. Prediction error for each sample for $Y_{1}, Y_{2}$, and $Y_{3}$ prediction. a) Porosity [\%], b) standard deviation [nm], c) diameter $[\mathrm{nm}]$.

Error $[\%]=$

$=\frac{\text { Experimental values }- \text { Predicted values }}{\text { Experimental values }} \cdot 100$

Granting to the findings of ANN modeling in the present survey, the three-dimensional surface plots were used to depict the relationships between input and output variables. Figures 8, 9 and 10 shows three-dimensional (3D) plots of the relationships between the influence of PVdF composition, solution concentration and voltage on nanofiber diameter, the standard deviation of nanofiber diameter, and porosity of electrospun membrane derived from the above model. In each figure, the effect of two input factors was studied on the obtained porosity, the standard deviation of nanofiber diameter and nanofiber diameter respectively, and the values of the remaining input factors were fixed at low, medium, and highrange value.

It is seen that there was no significant mutual interaction between these three parameters, and applied voltage has no important effect on nanofiber diameter.
The applied voltage may influence some parameters, such as electrostatic force, feed rate, the morphology of nanofibers, etc. The final diameter of the electrospun nanofibers is determined using a balance among these parameters. The applied voltage has two diverse effects on nanofiber diameter. First, by increasing the applied voltage, the electrostatic force increase and hereafter lead to the production of smaller diameter nanofibers. Second, increasing the applied voltage draws more solution out of the needle, per unit time, consequently increasing the diameter. If the first effect dominates, the nanofiber diameter will decrease with applied voltage. On the other hand, if the second effect dominates, nanofiber diameter increases with applied voltage.

The nanofiber diameter increased considerably with solution concentration, which implies the important role of solution concentration on nanofiber diameter. It has been reported that a minimum polymer concentration is required for electrospinning [36, 37]. Based on some of our preliminary experiments, we concluded that the critical concentration of PAN/PVdF 


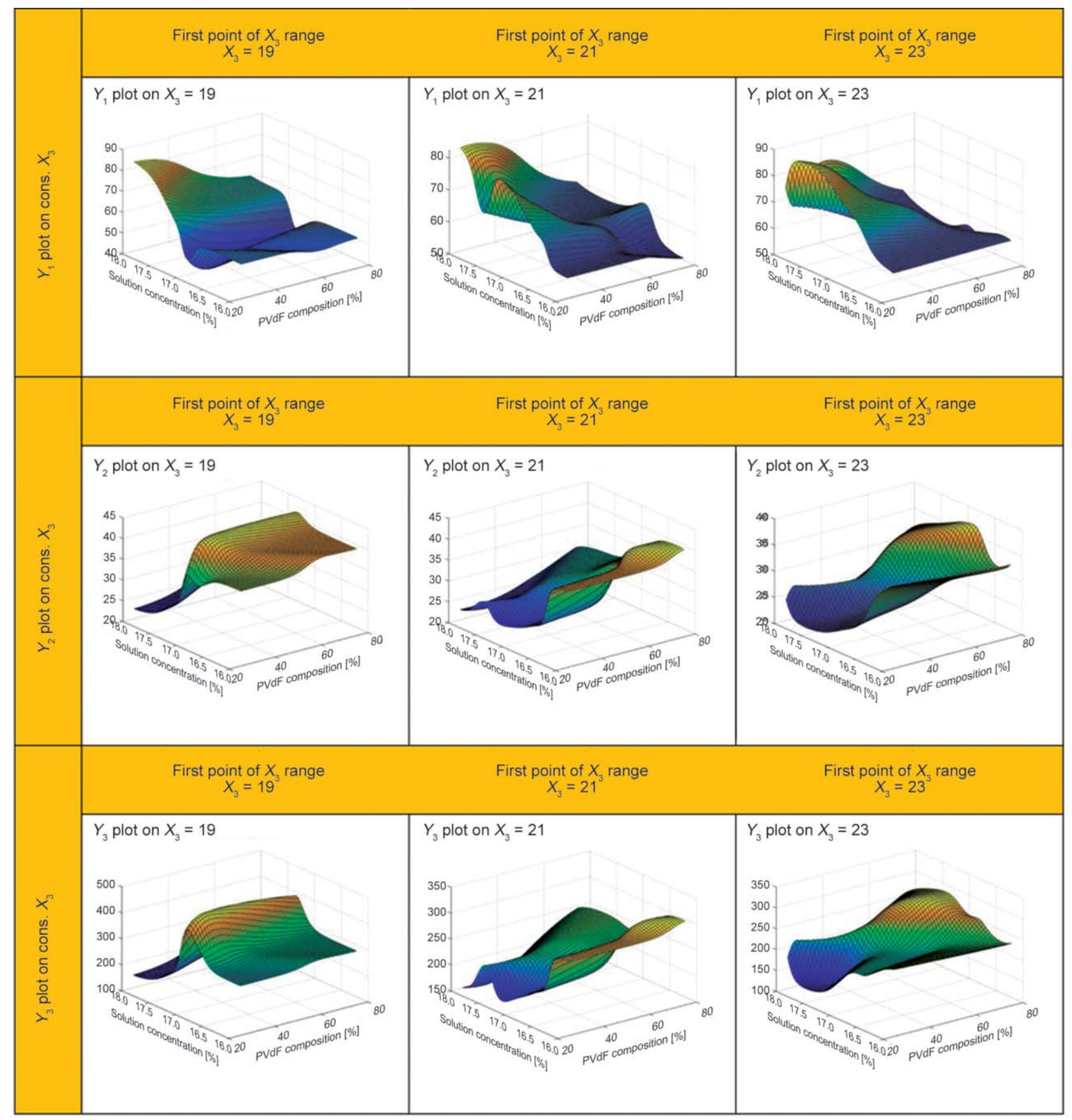

Figure 8. 3D plots of modeling responses (porosity $\left(Y_{1}\right)$, standard deviation of nanofiber diameter $\left(Y_{2}\right)$, and nanofiber diameter $\left.\left(Y_{3}\right)\right)$ in constant values of voltage.

solution in DMF for the development of continuous nanofibers was around $14 \%$. This concentration seems to be the beginning of chain entanglements in the PAN/PVdF solution. On the one hand, an increase in nanofiber diameter with solution concentration could be explained by competition between surface tension and viscosity [38]. Increasing the solution concentration leads to an increase in solution viscosity. At low solution concentration (low viscosity), surface tension overcomes viscoelastic force and tries to decrease surface area per unit mass; therefore, below a certain total polymer concentration, electrospraying will be attained instead of electrospinning. However, by increasing solution concentrations, electrospraying is reduced and finally eliminated; hence the viscoelastic force has a superior influence in comparison with surface tension and smooth and bead free nanofibers will be formed. On the other hand, solution with higher concentration consists of more polymer/unit volume of spinning solution; hence, the diameter would increase. Moreover, in electrospinning it would be hard to stretch a solution with higher viscosity and therefore thicker and nonuniform nanofibers will be produced. 


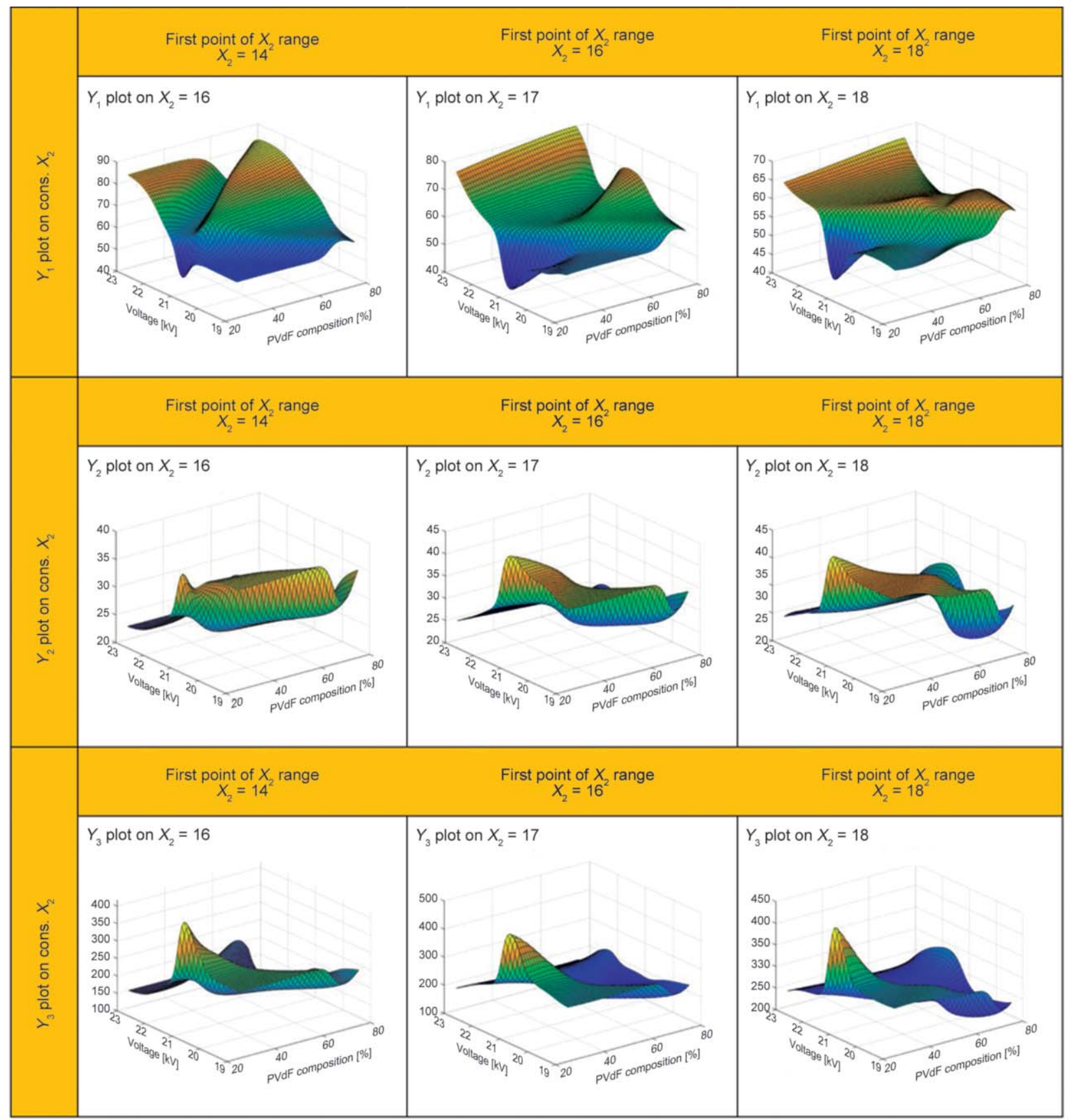

Figure 9. 3D plots of modeling responses (porosity $\left(Y_{1}\right)$, standard deviation of nanofiber diameter $\left(Y_{2}\right)$, and nanofiber diameter $\left.\left(Y_{3}\right)\right)$ in constant values of solution concentration.

It is observed that, at constant total polymer concentration, increasing PVdF composition leads to a decrease of nanofiber diameter. Since PVdF had lower solution viscosity than PAN at the same concentration, by increasing the PVdF content, the solution viscosity will decrease, which produces nanofiber with a smaller diameter.

The interlaying of fibers generates a porous structure in the electrospun membrane. In electrospun polymer electrolytes, porosity is one of the important parameters, since increasing the membrane porosity leads to an increase in contact areas between polymer and electrolyte. Consequently, the electrolyte will be well retained in the polymer. The fiber diameter has considerable effects on the physical properties of the membranes, such as porosity, pore size, pore size distribution, and specific surface area.

As shown in Figure 8, 9 and 10 porosity decreases with the fiber diameter. In electrospinning, a thinner solution jet results in the loose deposition of the fiber on the collector on account of the rapid evaporation of the solvent and hence leads to the increase in porosity. ANN is a mathematical pathway for process modeling. It should be denoted that, after process modeling, 


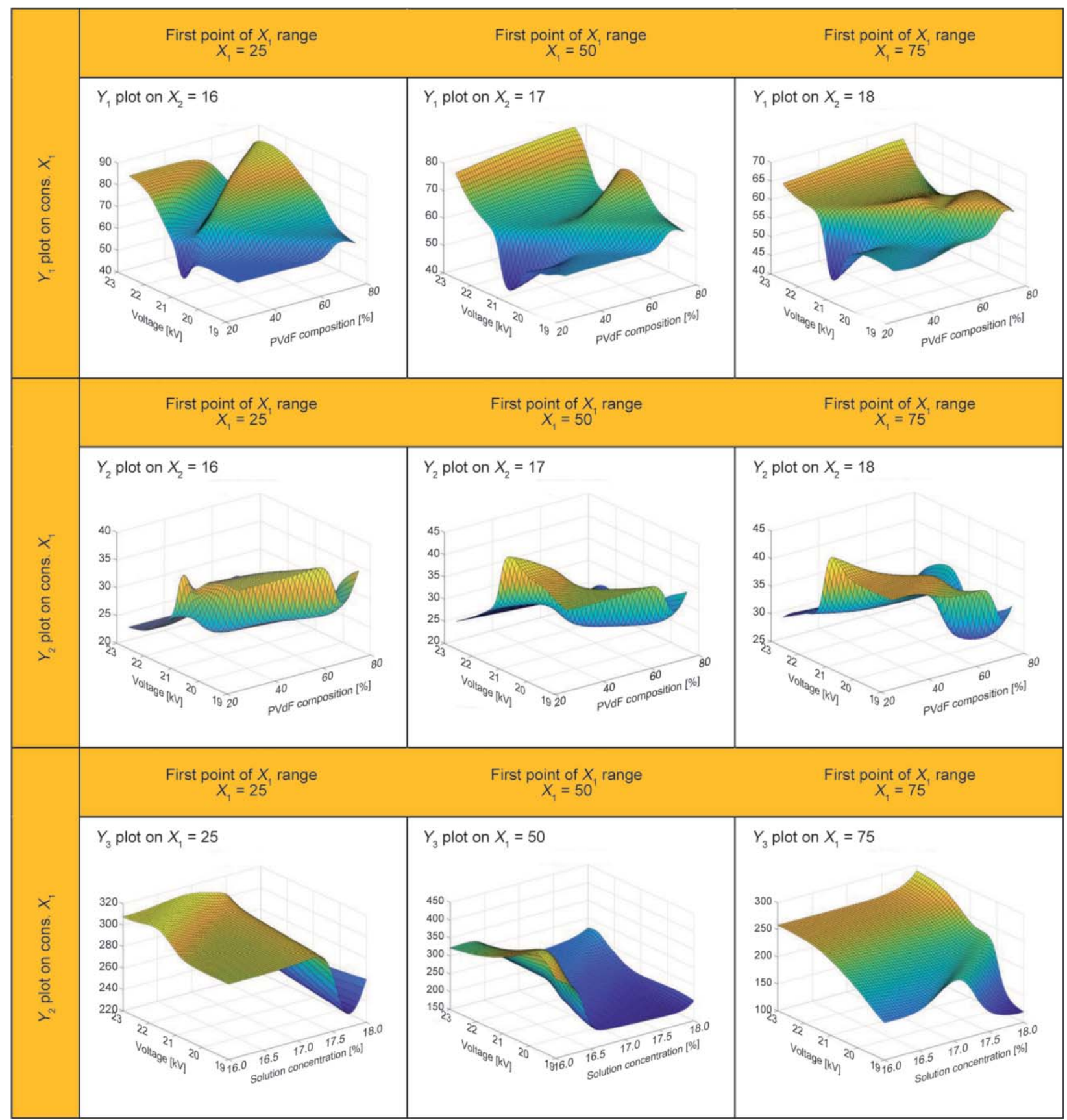

Figure 10. 3D plots of modeling responses (porosity $\left(Y_{1}\right)$, the standard deviation of nanofiber diameter $\left(Y_{2}\right)$, and nanofiber diameter $\left.\left(Y_{3}\right)\right)$ in constant values of PVdF composition.

an optimization method should be applied on ANN model for calculation of the best conditions for achieving favorite responses. Therefore, this study was continued by GA-based multi-objective optimization with NSGA II.

\subsection{Multi-objective optimization of PAN/PVdF nanofiber production based on ANN and generic algorithm}

In a wide range of processes, there is not a single point as the optimal solution with respect to all objectives. Therefore, a set of solutions are optimal points that each of them has one or more excellence over other solutions. This set of solutions has been known as Pareto optimal solution [39]. There is a wide range of classical manners and evolutionary algorithms for solving the MOO problems. MOO problems in classical methods have been solved by converting the MOO problem to a single objective optimization problem. In the other pathway of classical methods for MOO solving, MOO problem will be solved based on one object. In the following, other constraints will be applied to achieved solutions, and the best solutions will be chosen. Weighted sum 
method, e-constraint, and goal programming are some of the classical methods for MOO solving [39]. Changing the MOO problems to a single objective problem, usually lead to much easier solving and less computational cost. However, this method is not efficient for a wide range of problems [40]. On the other hand, there are several evolutionary algorithms to solve MOO problems, such as a genetic algorithm. Solving the Multi-Objective problems by Genetic Algorithm (MOGA) was introduced by Fonseca and Fleming firstly in 1993. There is a major difference between the MOGA and standard G.A. that is in ranking G.A. population (MOGA uses the non-dominated classification concept in the ranking of G.A. population) $[40,41]$. It is clear that MOGA can be used for any mathematical models to solve a multi-objective problem (of course, with a little change in circumstances). In this study, a trained ANN was used as a mathematical model fed to an evolutionary algorithm for optimizing the electrospun PAN/PVdF nanofiber properties.

To develop the models successfully, an effort was made to optimize PAN/PVdF nanofiber production (PVdF composition, solution concentration, and voltage) for a nanofiber diameter (minimize), the standard deviation of nanofiber diameter (minimize), porosity (maximize). These objects were searched by different amounts of inputs in a specified range, fed to a validated ANN model.

The Pareto optimal set for this object is presented in Figures 11 and 12. Plotting the corresponding decision variable against one of the objectives makes the understanding of their role in generating the optimal Pareto set possible.

As per results given in Table 3, this procedure of PAN/PVdF nanofiber production can produce nanofiber with a wide range of characteristics.

From the optimization results, if we wanted to consider the nanofiber diameter and our goal was producing nanofiber with minimum diameter according to optimization results, we can use this processing

Table 3. Detailed values for two-point with infinite crowding distance of $Y_{1}$ maximizing $-Y_{2}$ minimizing optimization (Case I) and $Y_{1}$ maximizing $-Y_{3}$ minimizing optimization (Case II) (other points are presented in supporting information).

\begin{tabular}{|l|c|c|c|c|c|}
\hline & $\boldsymbol{X}_{\mathbf{1}}$ & $\boldsymbol{X}_{\mathbf{2}}$ & $\boldsymbol{X}_{\mathbf{3}}$ & $\boldsymbol{Y}_{\mathbf{1}}$ & $\boldsymbol{Y}_{\mathbf{2}}$ \\
\hline Case I & 75.0000 & 14.0000 & 19.0000 & 103.1926 & 12.4989 \\
\hline & $\boldsymbol{X}_{\mathbf{1}}$ & $\boldsymbol{X}_{\mathbf{2}}$ & $\boldsymbol{X}_{\mathbf{3}}$ & $\boldsymbol{Y}_{\mathbf{1}}$ & $\boldsymbol{Y}_{\mathbf{2}}$ \\
\hline Case II & 75.0000 & 14.0000 & 19.5893 & 99.4169 & 82.2348 \\
\hline
\end{tabular}

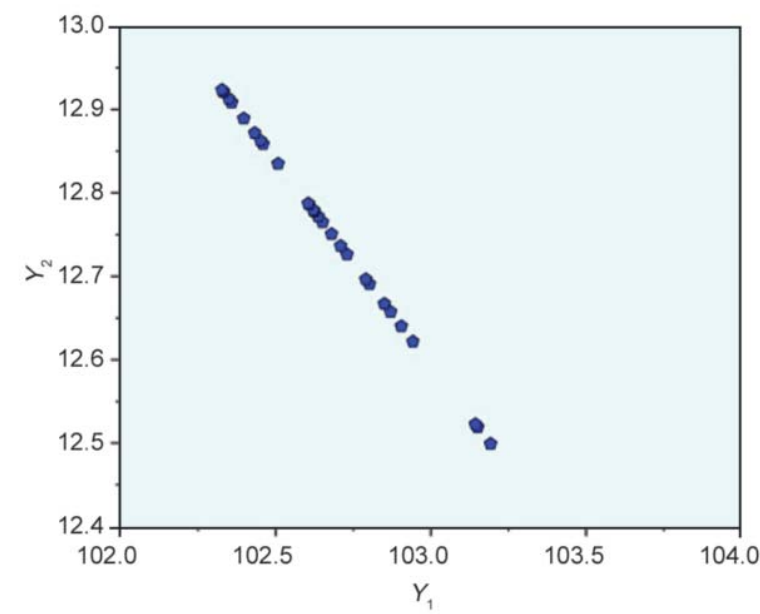

Figure 11. Pareto optimal front for maximizing porosity $\left(Y_{1}\right)$ and minimizing STD $\left(Y_{2}\right)$.

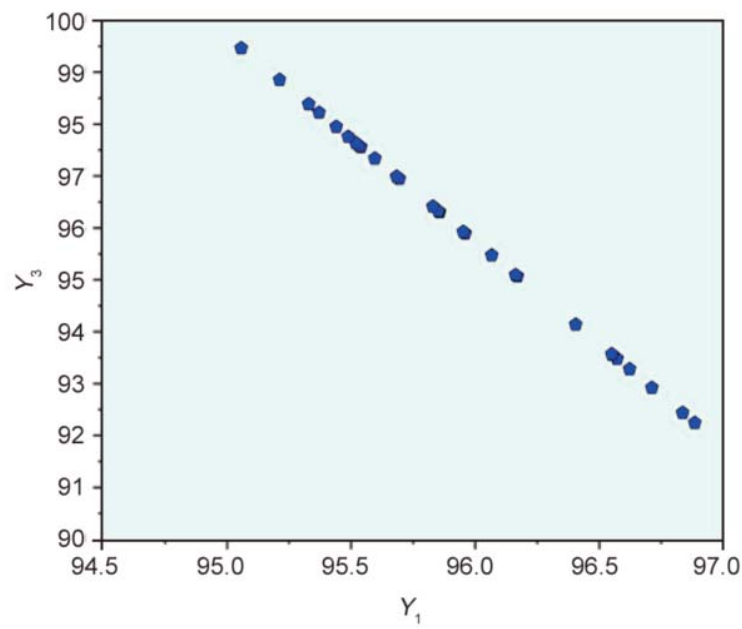

Figure 12. Pareto optimal front for maximizing porosity $\left(Y_{1}\right)$ and minimizing diameter $\left(Y_{3}\right)$.

condition $\left(X_{1}=75, X_{2}=14, X_{3}=19.5893\right)$. These processing conditions will lead to the production of nanofiber with $82.2348 \mathrm{~nm}$ diameter. In comparison with our previous work that we used RSM method to optimize the nanofiber diameter, using ANN method, we can reach nanofiber with less diameter (82.2348 versus 113.876$)$.

Furthermore, in nanofibrous polymer electrolyte, the porosity of membrane play an important part in electrolyte solution uptake and therefore in ionic conductivity. So if we wanted to maximize the porosity of nanofibrous membrane, we could use these processing conditions $\left(X_{1}=75, X_{2}=14, X_{3}=19\right)$. These processing conditions will lead to the production of nanofiber with $103.1926 \%$ porosity.

So, according to optimization results, the optimum processing condition to obtain PAN/PVdF nanofiber with minimum nanofiber diameter and maximum porosity is close to each other so we can conclude 
that in electrospinning of PAN/PVdF nanofiber decreasing the nanofiber diameter lead to higher porosity.

Furthermore, we can see that the simultaneous optimization of nanofiber diameter and standard deviation of nanofiber diameter has no considerable results.

\section{Conclusions}

In this study, PAN/PVdF nanofibers were produced using electrospinning. This process is a multi-objective problem and needs detailed modeling and optimization procedure for specifying the optimum conditions. A multi-layer perceptron artificial neural network modeling was used for process modeling, and NSGA II was applied on ANN model for optimization of electrospinning process variables (applied voltage, solution concentration, and PVdF composition) and achieve to suitable predict about physical characteristics of PAN/PVdF nanofiber. In ANN method, good fitness (with R higher than 95\%) was achieved, and it was also found that applied voltage has no significant effect on the responses. The nanofibers diameter and its standard deviation increase with increasing solution viscosity (increasing solution concentration and decreasing PVdF composition). The multi-objective optimization was done to estimate optimal conditions for the production of a $\mathrm{PAN} / \mathrm{PVdF}$ nanofiber with the minimum diameter and maximum porosity. On the other hand, the minimum values of nanofiber diameter lead to the best quality polymer electrolyte. Moreover, each of the other objects has a unique optimum level that should be considered in NSGA II. Finally, several points are reported to achieve these objectives.

\section{References}

[1] Doshi J., Reneker D. H.: Electrospinning process and applications of electrospun fibers. Journal of Electrostatics, 35, 151-160 (1995).

https://doi.org/10.1016/0304-3886(95)00041-8

[2] Huang Z-M., Zhang Y-Z., Kotaki M., Ramakrishna S.: A review on polymer nanofibers by electrospinning and their applications in nanocomposites. Composites Science and Technology, 63, 2223-2253 (2003). https://doi.org/10.1016/S0266-3538(03)00178-7

[3] Jian S., Zhu J., Jiang S., Chen S., Fang H., Song Y., Duan G., Zhang Y., Hou H.: Nanofibers with diameter below one nanometer from electrospinning. RSC Advances, 8, 4794-4802 (2018).

https://doi.org/10.1039/C7RA13444D
[4] Duan G., Greiner A.: Air-blowing-assisted coaxial electrospinning toward high productivity of core/sheath and hollow fibers. Macromolecular Materials and Engineering, 304, 1800669/1-1800669/5 (2019).

https://doi.org/10.1002/mame.201800669

[5] Li D., Xia Y.: Electrospinning of nanofibers: Reinventing the wheel? Advanced Materials, 16, 1151-1170 (2004). https://doi.org/10.1002/adma.200400719

[6] Kong C. S., Yoo W. S., Jo N. G., Kim H. S.: Electrospinning mechanism for producing nanoscale polymer fibers. Journal of Macromolecular Science Part B: Physics, 49, 122-131 (2010). https://doi.org/10.1080/00222340903344390

[7] Jiang S., Chen Y., Duan G., Mei C., Greiner A., Agarwal S.: Electrospun nanofiber reinforced composites: A review. Polymer Chemistry, 9, 2685-2720 (2018). https://doi.org/10.1039/C8PY00378E

[8] Greiner A., Wendorff J. H.: Electrospinning: A fascinating method for the preparation of ultrathin fibers. Angewandte Chemie International Edition, 46, 5670-5703 (2007). https://doi.org/10.1002/anie.200604646

[9] Ding C., Fang H., Duan G., Zou Y., Chen S., Hou H.: Investigating the draw ratio and velocity of an electrically charged liquid jet during electrospinning. RSC Advances, 9, 13608-13613 (2019). https://doi.org/10.1039/C9RA02024A

[10] Ding Y., Zhang P., Long Z., Jiang Y., Xu F., Di W.: The ionic conductivity and mechanical property of electrospun $\mathrm{P}(\mathrm{VdF}-\mathrm{HFP}) / \mathrm{PMMA}$ membranes for lithium ion batteries. Journal of Membrane Science, 329, 56-59 (2009).

https://doi.org/10.1016/j.memsci.2008.12.024

[11] Tan S-H., Inai R., Kotaki M., Ramakrishna S.: Systematic parameter study for ultra-fine fiber fabrication via electrospinning process. Polymer, 46, 6128-6134 (2005). https://doi.org/10.1016/j.polymer.2005.05.068

[12] Ribeiro C., Sencadas V. Gómez Ribelles J. L., LancerosMendez S.: Influence of processing conditions on polymorphism and nanofiber morphology of electroactive poly(vinylidene fluoride) electrospun membranes. Journal of Soft Materials, 8, 274-287 (2010). https://doi.org/10.1080/1539445X.2010.495630

[13] Areias A. C., Gómez-Tejedor J. A., Sencadas V., Alió J., Gómez Ribelles J. L., Lanceros-Mendez S.: Assessment of parameters influencing fiber characteristics of chitosan nanofiber membrane to optimize fiber mat production. Polymer Engineering and Science, 52, 12931300 (2012). https://doi.org/10.1002/pen.23070

[14] Prasanth R., Shubha N., Hng H. H., Srinivasan M.: Effect of nano-clay on ionic conductivity and electrochemical properties of poly(vinylidene fluoride) based nanocomposite porous polymer membranes and their application as polymer electrolyte in lithium ion batteries. European Polymer Journal, 49, 307-318 (2013). https://doi.org/10.1016/j.eurpolymj.2012.10.033 
[15] Lim D-H., Manuel J., Ahn J-H., Kim J-K., Jacobsson P., Matic A., Ha J. K., Cho K. K., Kim K-W.: Polymer electrolytes based on poly(vinylidene fluoride-co-hexafluoropropylene) nanofibrous membranes containing polymer plasticizers for lithium batteries. Solid State Ionics, 225, 631-635 (2012).

https://doi.org/10.1016/j.ssi.2012.03.028

[16] Raghavan P., Zhao X., Kim J-K., Manuel J., Chauhan G. S., Ahn J-H., Nah C.: Ionic conductivity and electrochemical properties of nanocomposite polymer electrolytes based on electrospun poly (vinylidene fluorideco-hexafluoroprooylene) with nano-sized ceramic fillers. Electrochimica Acta, 54, 228-234 (2008).

https://doi.org/10.1016/j.electacta.2008.08.007

[17] Duan G., Fang H., Huang C., Jiang S., Hou H.: Microstructures and mechanical properties of aligned electrospun carbon nanofibers from binary composites of polyacrylonitrile and polyamic acid. Journal of Materials Science, 53, 15096-15106 (2018). https://doi.org/10.1007/s10853-018-2700-y

[18] Zhou S., Zhou G., Jiang S., Fan P., Hou H.: Flexible and refractory tantalum carbide-carbon electrospun nanofibers with high modulus and electric conductivity. Materials Letters, 200, 97-100 (2017).

https://doi.org/10.1016/j.matlet.2017.04.115

[19] Duan G., Liu S., Hou H.: Synthesis of polyacrylonitrile and mechanical properties of its electrospun nanofibers. e-Polymer, 18, 569-573 (2018).

https://doi.org/10.1515/epoly-2018-0158

[20] Appetecchi G. B., Romagnoli P., Scrosati B.: Composite gel membranes: A new class of improved polymer electrolytes for lithium batteries. Electrochemical Communication, 3, 281-284 (2001).

https://doi.org/10.1016/S1388-2481(01)00137-0

[21] Raghavan P., Manuel J., Zhao X., Kim D-S., Ahn J-H., Nah C.: Preparation and electrochemical characterization of gel polymer electrolyte based on electrospun polyacrylonitrile nonwoven membranes for lithium batteries. Journal of Power Sources, 196, 6742-6749 (2011). https://doi.org/10.1016/j.jpowsour.2010.10.089

[22] Rao M., Geng X., Liao Y., Hu S., Li W.: Preparation and performance of gel polymer electrolyte based on electrospun polymer membrane and ionic liquid for lithium ion battery. Journal of Membrane Science, 399, 37 42 (2012).

https://doi.org/10.1016/j.memsci.2012.01.021

[23] Yoon H-K., Chung W-S., Jo N-J.: Study on ionic transport mechanism and interactions between salt and polymer chain in PAN based solid polymer electrolytes containing $\mathrm{LiCF}_{3} \mathrm{SO}_{3}$. Electrochimica Acta, 50, 289-293 (2004).

https://doi.org/10.1016/j.electacta.2004.01.095

[24] Hakkak F., Rafizadeh M.: Optimization of electrospun polyacrylonitrile/poly(vinylidene fluoride) nanofiber diameter using the response surface method. Journal of Macromolecular Science Part B: Physics, 52, 12501264 (2013).

https://doi.org/10.1080/00222348.2013.763569
[25] Hosseinnezhad M., Saeb M. R., Garshasbi S., Mohammadi Y.: Realization of manufacturing dye-sensitized solar cells with possible maximum power conversion efficiency and durability. Solar Energy, 149, 314-322 (2017).

https://doi.org/10.1016/j.solener.2016.11.011

[26] Hosseinnezhad M., Shadman A., Rezaee B., Mohammadi Y., Saeb M. R.: Tandem organic dye-sensitized solar cells: Looking for higher performance and durability. Photonics and Nanostructures-Fundamentals and Applications, 31, 34-43 (2018).

https://doi.org/10.1016/j.photonics.2018.05.005

[27] Afarani H. T., Sadeghi M., Moheb A., Esfahani E. N.: Optimization of the gas separation performance of polyurethane-zeolite $3 \mathrm{~A}$ and ZSM-5 mixed matrix membranes using response surface methodology. Chinese Journal of Chemical Engineering, 27, 110-129 (2019).

https://doi.org/10.1016/j.cjche.2018.03.013

[28] Li X., Cheruvally G., Kim J-K., Choi J-W., Ahn J-H., Kim K-W., Ahn H-J.: Polymer electrolytes based on an electrospun poly(vinylidene fluoride-co-hexafluoropropylene) membrane for lithium batteries. Journal of Power Sources, 167, 491-498 (2007).

https://doi.org/10.1016/j.jpowsour.2007.02.032

[29] Ojha V. K., Abraham A., Snášel V.: Metaheuristic design of feedforward neural networks: A review of two decades of research. Engineering Applications of Artificial Intelligence, 60, 97-116 (2017).

https://doi.org/10.1016/j.engappai.2017.01.013

[30] Gholamian M. R., Ghomi S. M. T., Ghazanfari M.: A hybrid intelligent system for multiobjective decision making problems. Computers and Industrial Engineering, 51, 26-43 (2006).

https://doi.org/10.1016/j.cie.2006.06.011

[31] Guo Z., Zhao W., Lu H., Wang J.: Multi-step forecasting for wind speed using a modified EMD-based artificial neural network model. Renewable Energy, 37, 241249 (2012).

https://doi.org/10.1016/j.renene.2011.06.023

[32] Ataeefard M., Sadati Tilebon S. M., Saeb M. R.: Intelligent modeling and optimization of emulsion aggregation method for producing green printing ink. Green Processing and Synthesis, 8, 703-718 (2019). https://doi.org/10.1515/gps-2019-0041

[33] Xu Y., Zhu Y., Xiao G., Ma C.: Application of artificial neural networks to predict corrosion behavior of $\mathrm{Ni}-\mathrm{SiC}$ composite coatings deposited by ultrasonic electrodeposition. Ceramics International, 40, 5425-5430 (2014). https://doi.org/10.1016/j.ceramint.2013.10.125

[34] Hekmatjoo N., Ahmadi Z., Afshar Taromi F., Rezaee B., Hemmati F., Saeb M. R.: Modeling of glycolysis of flexible polyurethane foam wastes by artificial neural network methodology. Polymer International, 64, 11111120 (2015). https://doi.org/10.1002/pi.4873 
[35] Hosseinnezhad M., Shadman A., Saeb M. R., Mohammadi Y.: A new direction in design and manufacture of $c o$-sensitized dye solar cells: Toward concurrent optimization of power conversion efficiency and durability. Opto-Electronics Review, 25, 229-237 (2017). https://doi.org/10.1016/j.opelre.2017.06.003

[36] McKee M. G., Wilkes G. L., Colby R. H., Long T. E.: Correlations of solution rheology with electrospun fiber formation of linear and branched polyesters. Macromolecules, 37, 1760-1767 (2004).

https://doi.org/10.1021/ma035689h

[37] Koski A., Yim K., Shivkumar S.: Effect of molecular weight on fibrous PVA produced by electrospinning. Materials Letters, 58, 493-497 (2004).

https://doi.org/10.1016/S0167-577X(03)00532-9

[38] Wang C., Cheng Y-W., Hsu C-H., Chien H-S., Tsou SY.: How to manipulate the electrospinning jet with controlled properties to obtain uniform fibers with the smallest diameter? - A brief discussion of solution electrospinning process. Journal of Polymer Research, 18, 111-123 (2011).

https://doi.org/10.1007/s10965-010-9397-1
[39] Farshad F., Iravaninia M., Kasiri M., Mohammadi T., Ivakpour J.: Separation of toluene/n-heptane mixtures experimental, modeling and optimization. Chemical Engineering Journal, 173, 11-18 (2011). https://doi.org/10.1016/j.cej.2011.07.018

[40] Ngatchou P., Zarei A., El-Sharkawi A.: Pareto multi objective optimization. in 'Proceedings of the $13^{\text {th }}$ International Conference on Intelligent Systems Application to Power Systems, Arlington, USA' 6-10 (2005). https://doi.org/10.1109/ISAP.2005.1599245

[41] Konak A., Coit D. W., Smith A. E.: Multi-objective optimization using genetic algorithms: A tutorial. Reliability Engineering and System Safety, 91, 992-1007 (2006).

https://doi.org/10.1016/j.ress.2005.11.018 